\title{
Vejez y radio en la pandemia: el caso de la Cadena SER y Radio Nacional de España
}

\section{Old age and radio in the pandemic: The case of Cadena SER and Radio Nacional de España}

\section{Velhice e rádio na pandemia: o caso Cadeia SER e Rádio Nacional de Espanha}

Virgina Guarinos, Universidad de Sevilla, Sevilla, España (guarinos@us.es)

Daniel Martin-Pena, Universidad de Extremadura, Badajoz, España (danielmartin@unex.es)

RESUMEN | Uno de los temas informativos generados de forma global por la crisis pandémica COVID-19 es el referente al contagio y fallecimiento de la población de más de 65 años. Dicho foco ha sido tratado en España por todos los medios de comunicación. En el marco de los Estudios etarios, esta investigación analiza la presencia y representación de la vejez en la radio española durante el confinamiento y la resignificación de un estereotipo de vejez activa que había sido sustituido en los medios informativos, la ficción y la publicidad en las dos últimas décadas por una imagen de la edad dorada relacionada con el envejecimiento activo. Se estudian las programaciones radiofónicas de la Cadena SER, la emisora privada con la mayor audiencia según el Estudio General de Medios, y de Radio Nacional de España durante cuatro días señalados del confinamiento: el día del inicio del estado de alarma en el país, el comienzo de la fase 0 de desconfinamiento y otros dos días intermedios relacionados con la emergencia en las residencias de mayores. Se analiza un total de 14 programas, 41 ediciones y 118 horas de radio. Los resultados muestran la existencia de contenidos de edadismo positivo, tanto en la representación de la vejez, de nuevo anclada en la dependencia, la fragilidad, la enfermedad y la muerte, como en el nivel de presencia del tema.

PALABRAS CLAVE: edadismo; radio; COVID-19; estereotipos; periodismo radiofónico; estudios etarios; Cadena SER; Radio Nacional de España. 
ABSTRACT / One of the global topics generated by the pandemic crisis COVID-19 is the infection and death of the population over 65 years. In Spain, this focus has been approached by all the media, including radio. Within the framework of Ageing Studies, this research analyzes the representation and presence of old age on Spanish radio during the blockdown, and the resignification of a stereotype of old age that had been replaced in the news media, fiction, and advertising in the last two decades by an image of the golden age related to active aging. The radio programming of Cadena SER, a private station with the largest audience according to the General Media Study, and of Radio Nacional de España were studied during four specific days of confinement: the day of the start of the state of alarm in the country, the beginning of phase 0 of deconfinement and another two intermediate days related to the emergency in nursing homes. A total of 14 programs, 41 editions and 118 radio hours are analyzed. The results show the existence of positive ageism, both in the representation of old age, once again anchored to dependency, frailty, illness, and death, and in the level of presence of the subject.

KEYWORDS: ageism; Radio; COVID-19; stereotypes; radio journalism; ageing studies; Cadena SER; Radio Nacional de España.

RESUMO|Uns dos tópicos informativos gerados mundialmente pela crise pandêmica COVID-19 é o contágio e a morte da população acima dos 65 anos. Este enofque tem sido coberto na Espanha por todos os meios de comunicação. No âmbito dos estudos de etários, esta investigação analisa a presença e representação da velhice na rádio espanhola durante o confinamento e ressignificação de um estereótipo da velhice ativa que havia sido substituído na mídia, na ficção e na publicidade nas duas últimas décadas por uma imagem da idade dourada relacionada com o envelhecimento ativo. Esta investigação se realiza sobre a programação das rádios da Cadeia SER, emissora privada de maior audiência segundo oEstudo Geral de Mídia, e da Rádio Nacional de Espanha durante quatro dias importantes de confinamento: o dia do início do estado de alarme no país, o início da fase 0 de desconfinamento e dois outros dias intermediários relacionados à emergência em lares de idosos. Foram analisados 14 programas, 41 edições e 118 horas de rádio. Os resultados mostram a existência de uma discriminação por idade positiva, tanto na representação da velhice, novamente ancorada na dependência, a fragilidade, a doença e a morte, quanto ao nível de presença do tópico.

PALAVRAS-CHAVE: discriminação por idade; Rádio; COVID-19; estereótipos; jornalismo de rádio; estudos etários; Cadeia SER; Rádio Nacional de Espanha. 


\section{INTRODUCCIÓN}

A nivel global, la pandemia por COVID-19 ha afectado fuertemente a las personas mayores, el colectivo que registra mayor porcentaje de contagios y fallecimientos. En España, las personas mayores de 65 años constituyeron $60 \%$ de toda la población afectada por esta enfermedad en los primeros meses de desarrollo de la pandemia. Este hecho las ha situado en primera línea de información y comunicación, convirtiéndose en protagonistas involuntarios de numerosos titulares de prensa, noticias en informativos y programas de televisión.

Ante la situación de la crisis pandémica, la respuesta internacional de los investigadores en comunicación social ha sido inmediata, con la publicación de distintas investigaciones que abordan nuestro objeto de estudio. Coleman (2020), Fonseca (2020) o Rodero (2020) han publicado estudios desde la perspectiva del medio radiofónico sobre hábitos de consumo durante el confinamiento en Reino Unido, Cuba y España, respectivamente. Por su parte, autores como Meisner (2020) o Ayalon (2020), en cuanto al edadismo se refiere, han detectado la intensificación de la discriminación por edad y la tensión intergeneracional a raíz de la crisis generada por la pandemia de COVID-19. En este sentido, incluso las Naciones Unidas han alertado sobre este asunto afirmando que "necesitamos abordar la discriminación por edad y el estigma contra las personas mayores de frente" (United Nations, 2020, p. 4).

En España, la presencia de las noticias y referencias en torno al impacto de la COVID-19 sobre la vejez se ha hecho patente tanto en prensa como en televisión, pero no tanto en la radio. Es por ello que este estudio resulta de interés, para complementar otras investigaciones recientes sobre el tema, como las de Bravo Segal y Villar (2020) a propósito del edadismo creciente a partir de titulares de diarios, o de Montaña Blasco y sus colegas (2020) sobre el consumo de medios en España durante la pandemia, o su impacto sobre la televisión en Europa, como referencia el trabajo de Túñez-López y colaboradores (2020).

Pocos investigadores podían prever que la radio iba a cumplir su primer centenario de existencia demostrando una vez más que es un medio vivo y joven para afrontar y comunicar momentos de crisis (Rodero \& Blanco, 2020). Esta investigación busca observar qué ha sucedido en el caso de la radio convencional con respecto a este tema nuclear durante el primer periodo de la pandemia, en cuanto al abordaje de este colectivo en este medio de comunicación.

\section{MARCO TEÓRICO}

Una investigación sobre edadismo, pandemia y radio necesariamente debe contemplar dos perspectivas. Por un lado, la fundamentada en estudios etarios y, por otro, la de estudios en comunicación, que a su vez se despliega en estudios 
sobre el lenguaje radiofónico, el periodismo oral y la comunicación de crisis. Los estudios etarios en comunicación pueden ser abordados desde el punto de vista del análisis de la representación y los estereotipos, pero también desde la recepción. Esta investigación se enmarca en el primer caso.

Los estudios etarios no son muy numerosos en el entorno de la comunicación y de la información, pero cuentan con fundamentos teóricos e investigaciones aplicadas significativas. Dichos estudios conciernen a una parcela de los estudios culturales (Hareven, 1996), atendiendo a la representación y al tratamiento de los grupos humanos en función de la edad con la que son representados. En el caso de los estudios etarios vinculados con la comunicación, han sido materia preferente de estudio la infancia y la adolescencia, sobre todo en lo referido a la representación de estas edades en la publicidad y en las series de televisión, así como en el cine (Chivers, 2011; Gravagne, 2013; Dolan, 2017), además de en los productos audiovisuales y lúdicos (videojuegos, fundamentalmente, y canales de YouTube) destinados a niños, niñas y adolescentes, a los que además se les suele dar una perspectiva de género (Medina \& Zecchi, 2020). El colectivo de los mayores, sin embargo, ha sido uno de los más degradados en la desindividualización. Se puede considerar, por tanto, como lo hacen Gullette \& Molinario, "la edad como una herramienta para dividir categorías y determinar subjetividades" (2010, p. 79).

Hemos asistido en los últimos veinte años a la resignificación de todas las edades (Rey-Beltrán, 2004) y a estereotipar a la población mayor (Muñoz, 2005). Del concepto de tercera edad se ha pasado al de edad dorada, una edad sana, activa, plena, que queda representada fundamentalmente en la publicidad y en las series de televisión, construyendo un estereotipo de viejóvenes tan distante de la realidad social como promocionado por los propios medios de comunicación y la publicidad (Ramos-Soler \& Carretón-Ballester, 2012)

Ya desde la década de los 70, la teoría de la vitalidad etnolingüística profundiza en la importancia del rol de los medios como elemento de fortaleza social y de influencia de los grupos en la sociedad (Abrams \& Eveland, 2003). La vitalidad es una medida de la fuerza de un grupo en la sociedad; es decir, del nivel de representación demográfica, apoyo institucional y estatus de los cuales disfruta. El contenido de los medios sobre un grupo social, en ese sentido, puede considerarse como un indicador de la fuerza y de la influencia de ese grupo.

Dentro de esta parcela de estudios etarios, la perspectiva del edadismo se centra en la representación y consumo como personajes y como receptores (a modo de espectadores, consumidores o usuarios), en este caso de la tercera edad, de modo discriminatorio o vejatorio. La definición de edadismo o etarismo se resume como "mantenimiento de estereotipos o actitudes prejuiciosas hacia una persona 
únicamente por el hecho de ser mayor" (Losada-Baltar, 2004, p. 2). Si bien existe un edadismo positivo (no dañino), para algunos teóricos (Palmore, 1999) es poco común, siendo el edadismo negativo (Butler, 1980) el más desarrollado y resurgido en este siglo (Palmore, 2001; Gullette, 2011). Paradójicamente, el edadismo sería -junto con la discriminación por género y por raza- una de las tres grandes violencias, puesto que el de los jubilados es un sector deseado para el consumo, al menos en lo que a los medios se refiere, en un doble movimiento anti-age y pro-age (Iacub, 2008). En cuanto al medio radiofónico, autores como Aznar y sus colegas (2015) han abordado la importancia del medio con respecto a la representación del envejecimiento activo en contraste con la baja presencia del colectivo de mayores en los magazines matinales.

Asimismo, en palabras de Suárez-Serrano (2016), el periodismo y los medios de comunicación "se sitúan en el centro del debate: son a un tiempo herramientas para hacer las nuevas guerras y también valiosos aliados para paliar el sufrimiento de los inocentes" (p. 34). A este marco se debe añadir lo relativo al medio radiofónico, tanto en su capacidad como medio de servicio público en la comunicación de crisis como en lo concerniente a sus posibilidades expresivas. Bien es sabido que la radio da respuesta inmediata en situaciones extremas, incluida esta generada por el COvid 19; es un medio de alta credibilidad, quizás aún más ahora en la era de las fake news, para la comunicación de crisis globales, como lo ha demostrado Rodero (2020) en el caso de la radio española. Dicha credibilidad depende en buena medida de los contenidos, de los formatos y del uso de los elementos expresivos constituyentes del lenguaje radiofónico. Las funciones de la palabra, el silencio, la música y los efectos permiten poder realizar todo lo que se desee.

En dicho contexto, el objetivo de esta investigación es analizar el tratamiento radiofónico español otorgado a la población de más de 65 años, a partir del hecho producido durante la pandemia de COVID-19, en el que ese núcleo ha sido el más afectado por la enfermedad tanto en número de pacientes como de fallecimientos en el país. Se establece ese objetivo para demostrar la hipótesis de partida, que afirma que la radio convencional española ha tratado el tema de la vejez durante el periodo de confinamiento con edadismo, ya sea por ausencia o silenciamiento del tema o por subrepresentación. Como hipótesis secundaria se establece la demostración de un cambio de representación del sector poblacional de jubilados y personas mayores, pasando del último nuevo estereotipo de vejez activa a la recuperación del estereotipo de vejez negativa.

\section{METODOLOGÍA}

Se optó por una estrategia metodológica mixta de análisis de contenido, un enfoque mixto integrativo múltiple (Hernández et al., 2010), considerando que 
la multiperspectiva temática requiere complejidad de método, especialmente en estudios en comunicación, por lo que es la tendencia actual en investigación en comunicación audiovisual (Damasio \& López-Díez, 2020) a partir de las fases empleadas por Arbeláez y Onrubia (2014). En una primera instancia, se ha realizado un preanálisis que ha permitido determinar el corpus con base en criterios de temporalidad, audiencia, titularidad y programación de las emisoras. Los casos de estudio son la Cadena SER, la emisora privada generalista con mayor audiencia, según datos del último Estudio General de Medios (Neeo.es, 2020) correspondiente a la primera ola 2020, y Radio Nacional de España, la emisora pública de carácter nacional y con importantes índices de audiencia, siendo la cuarta emisora española generalista más escuchada. Asimismo, se determinaron cuatro días significativos en la horquilla temporal de la etapa de confinamiento en España, de lo que resultaron dos fechas clave para enmarcar el estudio: el 14 de marzo de 2020, fecha en la que se inicia el estado de alarma, y el 4 de mayo, cuando comienza la fase 0 de desescalada, o desconfinamiento progresivo, hacia la nueva normalidad y el final del estado de alarma. Entre ambos días existían dos otras dos fechas reseñables en el desarrollo del tema tratado: el 19 de marzo de 2020, día en el que aparece la noticia de la situación que se está viviendo en los geriátricos del país, con contagios y muertes masivas en todo el territorio, y 17 de abril, cuando la fiscalía general del Estado abre más de setenta investigaciones en residencias de mayores. En cuanto a la selección de los programas en cada emisora, se consideró como criterio de inclusión que fueran los con más audiencia de la franja de mañana, tarde, noche y madrugada, para que también tuvieran cabida los distintos formatos de programas y la diversidad de contenidos, desde la pura información hasta la radio más lúdica, de confidencias o la deportiva.

Con estos criterios, la muestra de la investigación queda compuesta por dos cadenas nacionales, una de carácter público y otra privada. Se analizó una media de siete programas representativos de cada una de las emisoras, lo que supone un corpus conformado por 14 programas, 41 ediciones y 118 horas de radio (tabla 1).

En la tabla 1, en la columna de voces, conviene puntualizar que P se refiere a profesionales (periodistas, comentaristas, contertulios/as y colaboradores habituales, que en su mayoría se encuentran en el rango de edad de entre 50 y 60 años) y NP hace referencia a no profesionales (testimonios, invitados/as, entrevistados/as, especialistas, oyentes, de edades mucho más variables, desde niñas y niños hasta personas muy mayores). 


\begin{tabular}{|c|c|c|c|c|c|c|}
\hline Cadena & Título & Franja & Conductores & Ediciones & Tiempo & Voces \\
\hline SER & Hoy por hoy & Mañana & Àngels Barceló & 3 & $19 \mathrm{~h}$ & 213 (P. 106/NP.107) \\
\hline SER & La ventana & Tarde & $\begin{array}{c}\text { Carles } \\
\text { Francino }\end{array}$ & 4 & $14 \mathrm{~h}$ & 148 (P.72/NP.76) \\
\hline SER & Hora 25 & Noche & Pepa Bueno & 4 & 14 h 30' & 178 (P.82/NP.96) \\
\hline SER & $\begin{array}{l}\text { A vivir que } \\
\text { son dos días }\end{array}$ & Mañana & Javier del Pino & 2 & $6 \mathrm{~h}$ & 66 (P.24/NP.42) \\
\hline SER & El larguero & Noche & Manu Carreño & 3 & $6 \mathrm{~h}$ & 46 (P.24/NP.22) \\
\hline SER & El faro & Madrugada & Mara Torres & 2 & $5 \mathrm{~h}$ & 68 (P.17/NP.51) \\
\hline SER & $\begin{array}{l}\text { Lavida } \\
\text { moderna }\end{array}$ & Madrugada & $\begin{array}{l}\text { David } \\
\text { Broncano }\end{array}$ & 2 & 1h $26^{\prime}$ & 8 (P.5/NP.3) \\
\hline RNE & $\begin{array}{l}\text { Las mañanas } \\
\text { de RNE }\end{array}$ & Mañana & $\begin{array}{c}\text { Îñigo Alfonso } \\
\text { y Pepa } \\
\text { Fernández }\end{array}$ & 3 & $21 \mathrm{~h}$ & 323 (P.132/NP.191) \\
\hline RNE & $\begin{array}{l}\text { No es un día } \\
\text { cualquiera }\end{array}$ & Mañana & Carles Mesa & 1 & $5 \mathrm{~h}$ & 32 (P.16/NP.16) \\
\hline RNE & $\begin{array}{l}\text { Solamente } \\
\text { una vez }\end{array}$ & Tarde & $\begin{array}{l}\text { Amaya Prieto y } \\
\text { Julio Valverde }\end{array}$ & 3 & 5 h $35^{\prime}$ & 55 (P.29/NP.26) \\
\hline RNE & El ojo crítico & Tarde & $\begin{array}{l}\text { Alberto } \\
\text { Martínez }\end{array}$ & 3 & $2 \mathrm{~h} 14^{\prime}$ & 20 (P.9/NP.11) \\
\hline RNE & 24 horas & Noche & $\begin{array}{l}\text { Antonio } \\
\text { Delgado }\end{array}$ & 3 & $6 \mathrm{~h} 25^{\prime}$ & 176 (P.74/NP.102) \\
\hline RNE & $\begin{array}{l}\text { Radiogaceta } \\
\text { deportes }\end{array}$ & Noche & Chema Abad & 3 & $1 \mathrm{~h} 17^{\prime}$ & 24 (P.13/NP.11) \\
\hline RNE & $\begin{array}{c}\text { Gente } \\
\text { despierta }\end{array}$ & Madrugada & $\begin{array}{l}\text { Alfredo } \\
\text { Menéndez }\end{array}$ & 2 & $7 \mathrm{~h}$ & 62 (P.28/NP.34) \\
\hline RNE & $\begin{array}{c}\text { Juntos paso a } \\
\text { paso* }\end{array}$ & Mañana & $\begin{array}{l}\text { Juan } \\
\text { Fernández }\end{array}$ & 4 & $3 \mathrm{~h} 34^{\prime}$ & 68 (P.9/NP.59) \\
\hline$\stackrel{2}{\text { cadenas }}$ & $\underset{\text { programas }}{14}$ & $\begin{array}{c}4 \\
\text { franjas }\end{array}$ & $\begin{array}{c}17 \\
\text { conductores }\end{array}$ & $\begin{array}{c}41 \\
\text { ediciones }\end{array}$ & 117 h 46' & $\begin{array}{c}1487 \\
\text { (P.640 / NP.847) }\end{array}$ \\
\hline
\end{tabular}

*no incluido en estudio

Tabla 1. Corpus pormenorizado

Fuente: Elaboración propia.

El reparto de horas y voces analizadas por cadena es el siguiente: Cadena SER, 65 h 56', 727 voces, y RNE, 51 h 25', 760 voces. La desigualdad de horas analizadas depende de la producción de los programas seleccionados en los días elegidos para la muestra. La Cadena SER emitió programas especiales en fin de semana, en este caso el sábado 14 de marzo. 
Una vez determinado el corpus teórico, en la fase descriptiva se establecieron las distintas categorías necesarias en el análisis multimodal exhaustivo, que recoja componentes de categorización a partir de tres dimensiones de análisis para cada programa, cada una de las cuales es expresada en una tabla: (1) por palabras clave, es decir, términos fundamentales implicados en el campo semántico en el que se centra el envejecimiento, fundamental para la conformación de ideas sobre aquello de lo que se habla o informa, (2) por tipo de pieza periodística y tiempo empleado, por su implicancia en el nivel de presencia -tanto por importancia como por dedicación al asunto-, (3) por recursos expresivos sonoros y no sonoros, y por su aporte a la derivación hacia la subjetividad, lingüísticos y no lingüísticos.

El proceso de captación de datos decidido inicialmente fue el de descarga y clasificación de programas por cadenas y una posterior audición tradicional (doble, una primera de acopio y una segunda de comprobación y depuración de duplicaciones y palabras clave descontextualizadas). Los datos se manejaron a través del software Atlas.ti.

\section{RESULTADOS}

\section{Cadena SER}

La Cadena SER arroja los siguientes resultados correspondientes a las categorías contempladas en nuestro análisis. En cuanto a las palabras clave (tabla 2) conformantes del campo semántico estudiado, se observa que en la designación a las personas en estudio la palabra clave más usada es mayor/es; en cuanto a los lugares, residencia/s, y sobre conceptos aparece solo aisladamente tercera edad. Relacionado con el campo semántico, en la edición del día 17 de abril de La ventana aparece la palabra gericultor.

A partir del número de apariciones de las palabras clave, es importante resaltar que los días en los que esta cadena hace más referencia al tema, con mayor aparición de palabras, son el 19 de marzo, primer día de difusión de la crisis de las residencias en España, y el 17 de abril, en el que la fiscalía abre investigaciones a estos centros. La proporción de presencia de este campo semántico en los días analizados es de 10\% (14 de marzo), 60\% (19 de marzo), 20\% (17 de abril) y 10\% (4 de mayo). Con respecto al tipo de voz que emplea otras palabras no mayoritarias, abuelo y anciano son usados preferentemente por las voces no profesionales (oyentes y entrevistados).

En lo que respecta al tipo de pieza periodística y al tiempo empleado (tabla 3 ), se denomina suelto al asunto investigado como tema colateral, o inserto en sumario de focos informativos en pandemia, o en referencias al tema en intervenciones breves de oyentes. 


\begin{tabular}{|c|c|c|c|c|c|c|c|c|}
\hline & 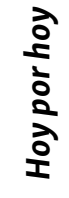 &  &  & 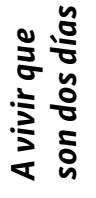 & 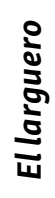 & $\frac{\frac{0}{5}}{\frac{1}{4}}$ & 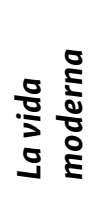 & $\begin{array}{l}\vec{\pi} \\
\stackrel{0}{\circ}\end{array}$ \\
\hline Mayor/es & 37 & 7 & 20 & 3 & 3 & 0 & 0 & $70(41 \%)$ \\
\hline Ancianos/as & 7 & 1 & 12 & 0 & 0 & 0 & 0 & $20(12 \%)$ \\
\hline Abuelos/as & 2 & 0 & 2 & 0 & 0 & 0 & 0 & 4 \\
\hline Viejo/a & 0 & 0 & 0 & 0 & 0 & 0 & 0 & 0 \\
\hline Residentes & 3 & 0 & 2 & 0 & 0 & 0 & 0 & 5 \\
\hline Jubilado/a & 0 & 2 & 1 & 0 & 0 & 1 & 0 & 4 \\
\hline Residencia/s & 21 & 6 & 30 & 0 & 0 & 0 & 0 & $57(33 \%)$ \\
\hline Centro & 2 & 0 & 4 & 0 & 0 & 0 & 0 & 6 \\
\hline Geriátrico & 4 & 0 & 0 & 0 & 0 & 0 & 0 & 4 \\
\hline Asilo & 0 & 0 & 0 & 0 & 0 & 0 & 0 & 0 \\
\hline Vejez & 0 & 0 & 0 & 0 & 0 & 0 & 0 & 0 \\
\hline Senectud & 0 & 0 & 0 & 0 & 0 & 0 & 0 & 0 \\
\hline Tercera edad & 0 & 1 & 0 & 0 & 0 & 0 & 0 & 1 \\
\hline
\end{tabular}

Tabla 2. Palabras clave. Cadena SER

Fuente: Elaboración propia.

Noticia desplegable es aquella información introducida por el presentador/a principal y desarrollada por un segundo periodista, que intercala testimonios de afectados o de especialistas en la materia. Junto con el tiempo empleado para tratar el tema, se establece un nivel de presencia que se califica como $0=$ ninguno; 1=bajo (de 1 a 30 minutos); $2=$ medio (de 30 a 60 minutos); $3=$ =alto (más de 60 minutos). La columna del tiempo empleado distingue por días analizados y el total global de cada programa.

Como sucede con las palabras clave, los días en los que más tiempo emplea la Cadena SER al tratamiento del tema son el 19 de marzo y el 17 de abril, siendo las franjas de mañana y de noche la que más tiempo le dedican y con mayor profundidad, al tratarse el tema en noticias desplegadas y en entrevistas. En el lado opuesto, la franja de madrugada no cuenta con presencia. Esta cadena, como cómputo global, prefiere el formato de sueltos (frases breves con información nuclear) y dedica 120 minutos, dos horas, de un total de 66 horas analizadas, lo que supone $1,32 \%$ del tiempo empleado en los cuatro días analizados. 


\begin{tabular}{|c|c|c|c|c|c|c|c|c|}
\hline & 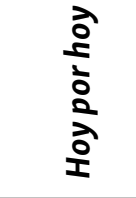 & 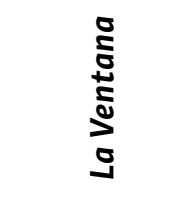 &  & 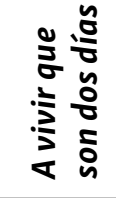 & 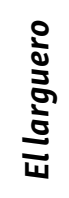 & $\frac{\frac{0}{0}}{\frac{5}{w}}$ & 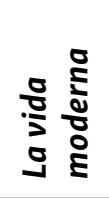 & $\begin{array}{l}\bar{\pi} \\
\stackrel{0}{0} \\
\end{array}$ \\
\hline Suelto & 21 & 3 & 11 & 2 & 1 & 0 & 0 & 38 \\
\hline Noticia & 4 & 2 & 4 & 0 & 0 & 0 & 0 & 10 \\
\hline $\begin{array}{c}\text { Noticia } \\
\text { desplegable }\end{array}$ & 2 & 1 & 3 & 0 & 0 & 0 & 0 & 6 \\
\hline $\begin{array}{l}\text { Tema de } \\
\text { tertulia }\end{array}$ & 1 & 0 & 1 & 0 & 0 & 0 & 0 & 2 \\
\hline $\begin{array}{l}\text { Tema de } \\
\text { entrevista }\end{array}$ & 1 & 4 & 1 & 0 & 2 & 0 & 0 & 8 \\
\hline $\begin{array}{l}\text { Tema de } \\
\text { editorial }\end{array}$ & 0 & 0 & 0 & 1 & 0 & 0 & 0 & 1 \\
\hline $\begin{array}{l}\text { Tiempo } \\
\text { dedicado }\end{array}$ & $42^{\prime} 15^{\prime \prime}$ & $31^{\prime} 23^{\prime \prime}$ & $43^{\prime} 07^{\prime \prime}$ & $2^{\prime} 20^{\prime \prime}$ & $1^{\prime}$ & 0 & 0 & $120^{\prime} 05^{\prime}$ \\
\hline Días & $19 / 17 / 4$ & $14 / 19 / 17 / 4$ & $14 / 19 / 17 / 4$ & 14 & 4 & 0 & 0 & Día 19 \\
\hline $\begin{array}{c}\text { Nivel } \\
\text { presencia }\end{array}$ & 2 & 2 & 2 & 1 & 1 & 0 & 0 & 2 \\
\hline
\end{tabular}

Tabla 3. Categorización de programas por tipo de pieza periodística y tiempo empleado. Cadena SER

Fuente: Elaboración propia.

El formato de suelto es el más abundante, al contrario de la presencia del tema como parte del editorial de cada programa, que solo cuenta con una presencia.

Los niveles expresivos de las voces y demás elementos sonoros y no sonoros se resumen en la tabla 4. Los efectos de radio hacen referencia a su presencia en el programa. En cuanto al estilo musical, se consignan las categorías informativa, culta, melancólica o de relleno. El silencio ha sido computado como la ausencia significativa de sonido. Por otra parte, se denomina adjetivación neutra a la estandarizada propia de la información (es decir, que no usa adjetivos calificativos ni sustantivos apreciativos), mientras que es expresiva aquella que demuestra subjetivización en el léxico utilizado (puede afectar también al tipo de términos utilizados, no adjetivos, como sustantivos en aumentativo o diminutivo, o palabras que por su propia carga semántica resultan hiperbólicas o de intensificación expresiva). Se registra también en la última fila de la tabla 4 , si la suma de los indicadores anteriores supone intencionalidad y sentido apreciativo. Además, se indica si corresponde a profesionales (P) o no profesionales (NP), siguiendo la nomenclatura usada para el reparto del tipo de voces analizadas previamente en la tabla. 


\begin{tabular}{|c|c|c|c|c|c|c|c|}
\hline & $\begin{array}{l}\text { वे } \\
\frac{1}{\vdots} \\
\vdots \\
\frac{0}{2} \\
\grave{1}\end{array}$ & 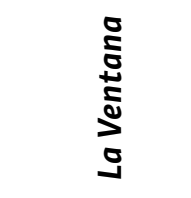 &  & 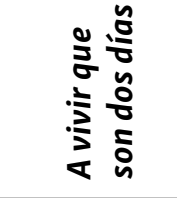 & 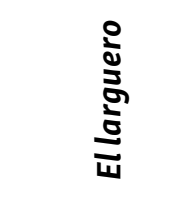 & $\frac{\frac{0}{0}}{\frac{1}{4}}$ & 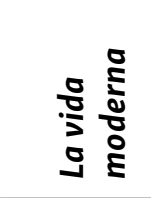 \\
\hline Efectos & No & No & No & No & No & No & No \\
\hline $\begin{array}{l}\text { Estilo } \\
\text { Musical }\end{array}$ & Informativa & Informativa & Informativa & Informativa & Informativa & Melancólica & Ninguna \\
\hline Prosodia & No & Sí & No & No & No & Sí & No \\
\hline Silencio & No & No & No & No & No & No & No \\
\hline Adjetivación & Neutra & $\begin{array}{c}\text { Neutra } \\
\text { Expresiva }\end{array}$ & $\begin{array}{l}\text { Neutra } \\
\text { Expresiva }\end{array}$ & No & $\begin{array}{c}\text { Expresiva } \\
\text { Neutra }\end{array}$ & Expresiva & Expresiva \\
\hline $\begin{array}{l}\text { Intención/ } \\
\text { sentido }\end{array}$ & NP & $P / N P$ & $P / N P$ & $P / N P$ & $\mathrm{P}$ & $P / N P$ & $P$ \\
\hline
\end{tabular}

\section{Tabla 4 . Niveles expresivos. Cadena SER}

Fuente: Elaboración propia.

\section{Radio Nacional de España}

El caso de Radio Nacional de España presenta una doble particularidad. Por un lado, es la única emisora que cuenta con un programa específico dedicado a este sector de la población, Juntos paso a paso; por otro, presenta una ruptura en la representatividad del tema investigado entre el primer día analizado y el resto, siendo muy notable una disparidad de criterios en los contenidos de la programación. A este respecto, es necesario aclarar que aunque el programa Juntos paso a paso ha sido analizado, los resultados no han sido incluidos en el cómputo general comparativo de cadenas, ya que tiene una audiencia modesta (se emite los sábados por la mañana, de 7:00 a 8:00, en una franja de emisión poco significativa) con respecto al resto de los programas analizados de ambas emisoras, que cuentan con audiencias importantes y son los espacios más destacados de cada una de las estaciones. Dicho programa solo emitió el primer día de las cuatro fechas seleccionadas para el corpus. En él tan solo aparece ocho veces la palabra "mayores", cuatro de ellas en declaraciones de un político y un médico, y otras cuatro entre los locutores, recomendando precaución a la población de dicha edad. "Centro de mayores" es referido una sola vez y, tan solo un oyente mayor interviene refiriéndose a las personas de su edad como "abuelos", en referencia a la soledad. Los profesionales del programa no utilizan recursos expresivos que subjetivicen la información, ni en sus voces ni en los recursos sonoros de música o efectos, dedicando su tiempo a otras cuestiones que no son de salud, como la economía plateada, un concurso de relatos o el aviso de suspensión de rutas culturales para este sector. Con respecto a lo segundo, cabe destacar que se trata de una política de toma de decisiones de la dirección del ente público. 
La intervención de Javier Hernández Bravo, director de RNE, en el Cuarto Conversatorio del I Encuentro Virtual de la RIU, celebrado telemáticamente el 18 de junio de 2020, deja clara dicha política. Además de mantener la programación al completo, se decidió dar voz a los oyentes a modo de desahogo en todos y cada uno de los programas y crear uno específico para ello, El balcón de Radio Nacional. En sus propias palabras, por miedo:

[La gente mayor] no quería oír hablar de pandemia, era una especie de recordatorio de que ellos podían morir en cualquier momento, entonces se desconectaban. Por lo tanto, el entretenimiento cobra un sentido tremendo. No es un contenido de segunda, todo lo contrario. Necesitábamos distraernos, necesitábamos distraerlos (...) Hay un programa que nosotros tenemos que se llama Juntos paso a paso. Está pensado, dirigido para la gente mayor. Es un programa de gran audiencia que se emite los sábados a primera hora y yo recuerdo el día en que hablé con su director y le dije: 'QQué vamos a hacer? Porque os tenéis que ir todos a casa'. Y dijo: 'Yo no voy a dejar a mis viejitos sin programa, entonces yo voy a hacer el programa como sea'. Y ese programa nunca faltó a su cita con contenidos nuevos" (Red de Radio Universitaria de Colombia, 2020, 1:14:20).

La desaparición de referencias hondas a la pandemia en relación con los mayores a partir del 19 de marzo queda justificada en buena medida por estas palabras, que denotan una toma de posiciones en la que la ausencia no ha sido por edadismo positivo sino por protección.

Como consecuencia, el uso de palabras clave queda muy reducido en esta emisora, concentrado en el día 19 de marzo. Aun así, muestra la misma tendencia que la Cadena SER, con un índice preferente para la palabra mayor/es y residencia, quedando ausentes las palabras referidas al concepto (tabla 5).

Existe una gran disparidad entre el número de voces y la cantidad de veces que las palabras clave aparecen en su contexto: 760 voces para 137 palabras. La palabra envejecimiento aparece solo dentro del programa dedicado a este sector de edad; otra denominación no presente en cualquier otro programa es senior, y otras no contempladas -consecuencia del tratamiento de temas no incluidos en el resto de programas y cadenas- son economía plateada o pensionista, jubilación, sistema de pensiones o jubilado, como categoría relacionada con lo laboral, tratada en profundidad y fuera del sintagma médicos jubilados, aparecido en otros programas al hacer referencia a la llamada de estudiantes de último año de medicina y médicos jubilados para cubrir la saturación de los hospitales. 

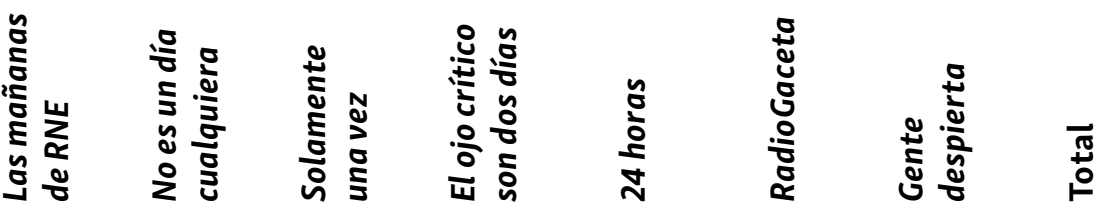

\begin{tabular}{ccccccccc}
\hline Mayor/es & 18 & 0 & 1 & 0 & 7 & 0 & 5 & $31(23 \%)$ \\
\hline Ancianos/as & 9 & 0 & 0 & 0 & 4 & 0 & 1 & $14(10 \%)$ \\
\hline Abuelos/as & 2 & 0 & 0 & 0 & 0 & 0 & 2 & 4 \\
\hline Viejo/a & 0 & 0 & 0 & 0 & 0 & 0 & 0 & 0 \\
\hline Residentes & 5 & 0 & 0 & 0 & 0 & 0 & 0 & 5 \\
\hline Jubilado/a & 0 & 0 & 0 & 0 & 3 & 0 & 0 & 3 \\
\hline Residencia/s & 50 & 0 & 1 & 0 & 10 & 0 & 1 & $62(45 \%)$ \\
\hline Centro & 4 & 0 & 0 & 0 & 9 & 0 & 0 & $13(9 \%)$ \\
\hline Geriátrico & 1 & 0 & 0 & 0 & 4 & 0 & 0 & 5 \\
\hline Asilo & 0 & 0 & 0 & 0 & 0 & 0 & 0 & 0 \\
\hline Vejez & 0 & 0 & 0 & 0 & 0 & 0 & 0 & 0 \\
\hline Senectud & 0 & 0 & 0 & 0 & 0 & 0 & 0 & 0 \\
\hline Tercera edad & 0 & 0 & 0 & 0 & 0 & 0 & 0 & 0 \\
\hline
\end{tabular}

Tabla 5. Palabras clave. RNE

Fuente: Elaboración propia.

Sobre las 49 horas de RNE, sin considerar el programa Juntos paso a paso, el tiempo dedicado, 59 minutos, supone un 0,28\% del tiempo analizado. El nivel de presencia es bajo, contando con dos programas en los que nunca se trata el tema. Se observa que el suelto tiene el mismo número de empleo que la suma del resto de posibilidades de formato contempladas para el análisis, concentrándose además en el día 19 de marzo (tabla 6).

Los niveles de expresividad de RNE quedan reducidos al mínimo, no solo en la utilización de recursos sonoros como la música, los efectos o el silencio, sino también con respecto al uso de la voz y la palabra (tabla 7). 

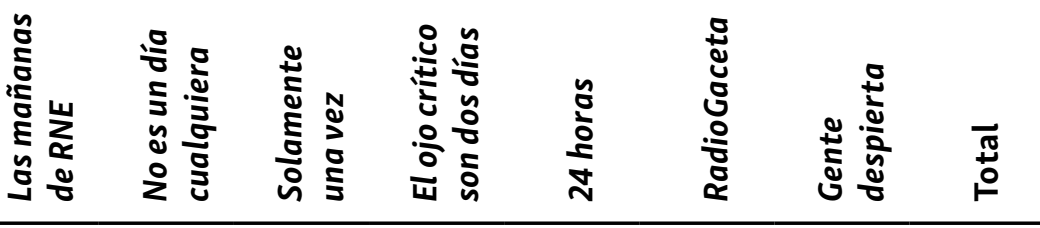

\begin{tabular}{ccccccccc}
\hline Suelto & 6 & 0 & 5 & 0 & 5 & 1 & 1 & 18 \\
\hline Noticia & 2 & 0 & 2 & 0 & 2 & 0 & 1 & 5 \\
\hline Noticia desplegable & 5 & 0 & 2 & 0 & 2 & 0 & 0 & 7 \\
\hline Tema de tertulia & 1 & 0 & 0 & 0 & 0 & 0 & 0 & 1 \\
\hline Tema de entrevista & 2 & 0 & 1 & 0 & 1 & 0 & 0 & 3 \\
\hline Tema de editorial & 0 & 0 & 1 & 0 & 1 & 0 & 0 & 2 \\
\hline Tiempo dedicado & $44^{\prime} 45^{\prime \prime}$ & 0 & $1^{\prime}$ & 0 & $11^{\prime} 55^{\prime \prime}$ & $15^{\prime \prime}$ & $1^{\prime} 30^{\prime \prime}$ & $58^{\prime} 45^{\prime \prime}$ \\
\hline Días & $19 / 17 / 4$ & - & $19 / 17$ & - & $19 / 17$ & 19 & $1 / 4$ & \\
\hline Nivel presencia & 2 & 0 & 1 & 0 & 1 & 0 & 1 & 1 \\
\hline
\end{tabular}

Tabla 6. Categorización de programas por tipo de pieza periodística y tiempo empleado. RNE

Fuente: Elaboración propia.

\begin{tabular}{|c|c|c|c|c|c|c|}
\hline  & 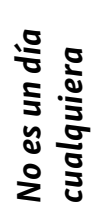 & 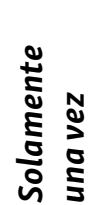 & 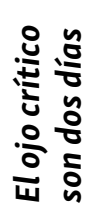 & $\frac{\tilde{c}}{\frac{\delta}{d}} \frac{d}{d}$ & 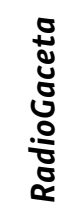 & 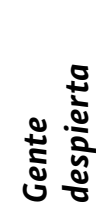 \\
\hline No & No & No & No & No & No & No \\
\hline
\end{tabular}

Estilo Informativa Informativa Relleno Culta Informativa Informativa Relleno

\begin{tabular}{cccccccc}
\hline Prosodia & Sí & No & No & No & No & No & No \\
\hline Silencio & No & No & No & No & No & No & No \\
\hline Adjetivación & $\begin{array}{c}\text { Expresiva } \\
\text { Neutra }\end{array}$ & Neutra & No & No & $\begin{array}{c}\text { Neutra } \\
\text { Expresiva }\end{array}$ & Neutra & No \\
\hline $\begin{array}{c}\text { Intención/ } \\
\text { Sentido }\end{array}$ & P / NP & NP & NP & No & P & NP & No \\
\hline
\end{tabular}

Tabla 7. Niveles expresivos. RNE

Fuente: Elaboración propia.

\section{Discusıón}

En el plano del análisis cualitativo, podemos señalar que, en la franja de mañana, en el programa Hoy por hoy de la Cadena SER los periodistas y colaboradores habituales 
rara vez incurren en subjetividad expresiva, si bien la información está asociada con el campo semántico de la muerte y la decrepitud, refiriéndose a las residencias como puntos negros o lugares críticos donde los enfermos acaban muriendo solos. Los oyentes de este programa, de todas las edades (incluidos niños y niñas) y sexos, son los que utilizan las palabras abuelo/a, anciano/a y adjetivos expresivos subjetivos, además de algún diminutivo apreciativo, por ejemplo: un poco sordito. Algunos políticos entrevistados y responsables de centros geriátricos también recurren a este tipo de lenguaje más emotivo. La música, los efectos y el silencio carecen de función en este programa. No existe diferencia en el uso expresivo o aséptico del lenguaje entre voces masculinas o femeninas, ya sean profesionales o no profesionales. Tampoco sucede con el factor de edad, igual que en el resto de los programas de la emisora. Por su parte, en Las mañanas de RNE, emisión en la que el tema tiene mayor número de apariciones, sobre todo en la primera parte de la mañana, de 6:00 a 10:00, es raro encontrar alguna expresión subjetiva. Se reitera la aparición de términos intensivos como virulencia, angustia, fallecimiento, dependencia, vulnerabilidad. El 17 de abril se pasa a hablar poco y de forma aséptica, sin implicaciones emocionales, denotando un cambio radical de actitud de la emisora, que fluctuó desde la implicación emocional hacia el distanciamiento de forma abrupta.

En la franja de tarde, en La ventana de la Cadena SER se oye hablar sobre la gente mayor como la franja de edad con la que se está cebando el virus, el colectivo más castigado o el colectivo más vulnerable y damnificado de la pandemia, recurriendo a un léxico menos subjetivo y más estandarizado en la información. Su conductor es el que más utiliza un tono cariñoso paternal, especialmente en las entrevistas a personas mayores. No obstante, es un epidemiólogo entrevistado el que aporta, el 4 de mayo, la utilización de superlativos, como mortalidad altísima. Cabe destacar que, siendo un programa con secciones humorísticas que pueden llegar a bromear con algunos temas pandémicos, nunca lo hacen con este en particular. Los oyentes mayores se refieren a sí mismos como mayores o expresando su edad y no hacen referencia a la salud sino a la soledad y al miedo. Por su parte, en RNE, en el programa Solamente una vez, se dice el día 19 de marzo: "Un saludo a los padres y a los que estéis solos y aislados en una residencia" (Prieto, 2020a), mientras que el 17, la presentadora empieza así el programa, evidenciando un cambio de política: "Como siempre, intentaremos no hacer mucho ruido y acompañarte con tranquilidad” (Prieto, 2020b).

En la franja de noche el programa Hora 25 de la SER retoma el tratamiento frío y distante de la franja de mañana, basado en cifras y escasa expresividad, evitando apreciaciones. La directora se expresa así, con rigor informativo: " 50 personas han muerto en residencias de mayores” (Bueno, 2020a), añadiendo a lo sumo los adjetivos vulnerables y ancianos, aunque puede añadir una intensificación 
expresiva para poner de manifiesto la magnitud del problema: la alarma generada por las muertes en las residencias de mayores, residencias desbordadas o personas mayores como grandes dependientes. La mayor parte de los recursos expresivos se concentran en las voces de oyentes en el programa del 19 de marzo, en el que muchos felicitaban a sus padres hospitalizados o en residencias. La expresividad más marcada se produce el 4 de mayo en la voz de una de las contertulias que expresa: "Más de la mitad de las personas muertas por el coronavirus estaban en residencias de ancianos, por favor... ¿Nadie está hablando de hacer una auditoría sobre esto? ¿Nadie está hablando de hacer un plan para que las residencias de ancianos no sean lo que han sido durante estos dos meses? El Gobierno debe buscar soluciones para que sean medicalizadas, para que no sean sitios para ganar dinero, que tiene una función social" (Méndez, 2020). Con tono severo y enfatizando las palabras, la única respuesta que encontró fue la de la conductora diciendo: "Debería ser el único tema político” (Bueno, 2020b), cerrando el tema y no profundizando. El cambio de tono también se percibe en 24 horas de RNE. El presentador dice el 19 de marzo: "Los mayores, los más vulnerables, y entre los más vulnerables los que están en residencias geriátricas. Allí han fallecido ya al menos 79 ancianos. La guardia civil está revisándolas para ver cuál es su situación (...) La situación de alguno de ellos ha sido un auténtico escándalo" (Delgado, 2020a). El 17 de abril el distanciamiento y la brevedad se resumen en este modo de narrar: "La Fiscalía General del Estado ha informado que tiene abierta al menos 38 investigaciones penales, 19 en Madrid, a residencias de mayores por la gestión de esta pandemia. Precisamente VOX ha presentado esta tarde una denuncia frente a la fiscalía provincial para que se investigue lo ocurrido en estos centros" (Delgado, 2020b).

Por último, cabe reseñar dos elementos más en la Cadena SER, dos casos de edadismo. En el A vivir que son dos días, del 14 de marzo, uno de los periodistas, tratando sobre un personaje de una novela dice de forma despectiva voz de vieja. El adjetivo viejo/a no se usa nunca en esta cadena para referirse a personas reales en temas vinculados con la pandemia; en este caso se utiliza para un personaje de ficción. En El larguero, siendo casi la única referencia al asunto en este programa, el 4 de mayo, un joven deportista se queja de haber sido increpado como irresponsable al salir a correr por parte de una pareja de personas mayores, a lo que el director Manu Carreño apostilla: “Claro... Te dirían cómo son los jóvenes...” (Carreño, 2020). También hay que apuntar que existe una presencia importante en el ámbito de la publicidad. Los días 14 y 19 de marzo cuentan con cuñas especiales de El Corte Inglés, ofreciendo la prioridad de compra y horarios como ayuda a los mayores para la compra en confinamiento, tuteando y dirigiéndose a un oyente mayor ( $E l$ Corte Inglés está a tu lado). El 17 de abril ya ha desaparecido esa cuña, y aparecido la de Cuideo, empresa destinada al cuidado de mayores. 


\section{CONCLUSIONES}

Más allá de estos ejemplos específicos, una abstracción de los datos lleva a concluir que:

- La representación de la vejez se realiza como estereotipo colectivo no individualizado, construido de forma contradictoria con base en eufemismos que eviten la negatividad semántica, pero reforzando la idea de dependencia, enfermedad y muerte. Esto supone una resignificación con respecto al último estereotipo de mayor alcance en las últimas décadas vinculado con la edad dorada (Guarinos, 2021).

- La presencia de la vejez es mínima en tiempo dedicado, pero también en profundización de los temas que le conciernen, debido a los formatos informativos menores y breves en los que se han vehiculado las noticias referidas a la ancianidad en pandemia.

Tanto los modos de representación como su presencia hablan en favor de un edadismo positivo, no hiriente, pero sí marginador, por la escasez de tiempo dedicado y por la poca profundidad en el tratamiento de los temas que afectan a las edades avanzadas. Dados los resultados, se infiere que esta investigación ratifica lo ya descubierto con respecto a otros medios de comunicación, en este caso en lo que a periodismo radiofónico se refiere, ofreciendo un panorama más completo, al sumar al medio radiofónico a los estudios etarios en comunicación, añadiendo además la confirmación de una brecha generacional producida por la crisis pandémica. Asimismo, este trabajo permite abrir nuevas líneas de estudio a partir del análisis de la programación de otras emisoras de cobertura nacional y su posterior comparación. No obstante, lo realmente interesante sería poder establecer la comparativa con otras radios internacionales de habla hispana, de modo que pueda lograrse un panorama más completo que ayude a superar esta limitación de parcialidad, al ser este un estudio reducido.

\section{REFERENCIAS}

Abrams, J. R. \& Eveland, W. P. (2003). The Effects of Television on Group Vitality: Can Television Empower Nondomint Groups? Annals of The Internacional Communication Association, 27(1), 193-219. https://doi.org/10.1080/23808985.2003.11679026

Arbeláez, M. C. \& Onrubia, J. (2014). Análisis bibliométrico y de contenido. Dos metodologías complementarias para el análisis de la revista colombiana Educación y Cultura (Bibliometric and Content Analysis. Two Complementary Methodologies for the Analysis Of the Colombian Magazine 'Education and Culture'). Revista de Investigaciones UCM, 14(23), 14-31. https://doi.org/10.22383/ri.v14i1.5

Ayalon, L. (2020). There is nothing new under the sun: Ageism and intergenerational tension in the age of the COVID-19 outbreak. International Psychogeriatrics, 32 (10), 1221-1224.

https://doi.org/10.1017/S1041610220000575 
Aznar, H., Álvarez, A., \& Suay, A. (2016). Estudio sobre la contribución de la radio al envejecimiento activo. Fase inicial: análisis cuantitativo de la presencia de la vejez en los magazines de radio 1 (Study on the Contribution of the Radio to Active Ageing. Initial Phase: Quantitative Analysis of the Topic of Ageing on Radio Magazines). Estudios sobre el mensaje periodístico, 22(1), 143-162. https://doi.org/10.5209/rev_ESMP.2016.v22.n1.52586

Bravo Segal, S. \& Villar, F. (2020). La representación de los mayores en los medios durante la pandemia COVID-19: ¿hacia un refuerzo del edadismo? (Older people representation on the media during COVID-19 pandemic: A reinforcement of ageism?). Revista Española de Geriatría y Gerontología, 55(5), 266-271. https://www.sciencedirect.com/science/article/ pii/S0211139X20300901

Bueno, P. (2020a, March 19). Hora 25 (radio broadcast). Cadena SER. https://play.cadenaser.com/audio/cadenaser_hora25_20200319_220000_230000/

Bueno, P. (2020b, May 4). Hora 25 (radio broadcast). Cadena SER. https://play.cadenaser.com/audio/cadenaser_hora25_20200504_210000_220000/

Butler, R. W. (1980). The Concept of a Tourist Area Cycle of Evolution: Implications for Management of Resources. Canadian Geographer, 24(1), 5-12. https://doi.org/10.1111/j.1541-0064.1980.tb00970.x

Carreño, M. (2020, May 4). El larguero (radio broadcast). Cadena SER. https://play.cadenaser.com/audio/1588547271399/

Chivers, S. (2011). The Silvering Screen. University of Toronto Press.

Coleman, J. F. (2020). UK Community Radio Production Responses to COVID-19. Retrieved from https://www.brunel.ac.uk/research/Documents/Jo-Coleman-UK-Community-RadioProduction-Responses-to-COVID-19.pdf

Damasio, M.J. \& López-Díez, J. (2020). Reflexión sobre un punto de inflexión en las metodologías de investigación en comunicación audiovisual (A reflection on a turning point in research methodologies on audiovisual Communication). Communication \& Methods, 2(1), 4-6. https://doi.org/10.35951/v2i1.73

Delgado, A. (2020a, March 19). 24 horas (radio broadcast). Radio Nacional de España. https:// www.rtve.es/play/audios/24-horas/analisis-informacion-03-11-2021/6177459/

Delgado, A. (2020b, April 17). 24 horas (radio broadcast). Radio Nacional de España. https://www.rtve.es/play/audios/24-horas/24-horas-analisis-informacion-17-04-20/5559871/

Dolan, J. (2017). Contemporary Cinema and 'Old Age': Gender and the Silvering of Stardom. Palgrave MacMillan.

Fonseca, B. E. (2020). La radio cubana ante la COVID-19. Un estudio de caso (The Cuban radio against the COVID-19. A case study). Revista española de Comunicación en Salud, 272278. https://doi.org/10.20318/recs.2020.5421

Gravagne, P. (2013). The Becoming of Age: Cinematic Visions of Mind, Body and Identity in Later Life. McFarland and Company, Inc. Publishers.

Guarinos, V. (2021). La vieja radio del salón. La programación radiofónica española hecha por y para mayores de 65 años (The old radio in the living room. Spanish radio programming made by and for people over 65). In V. Guarinos (Coord.), La isla etaria. Tercera edad $y$ medios de comunicación (The age island. Seniors and the media) (pp. 19-34). Readuck. 
Gullette, M. M. (2011). Agewise: Fighting the New Ageism in America. University of Chicago Press.

Gullette, M. M. \& Molinari, A. (2010). Los estudios etarios como estudios culturales. Más allá del slice-of-life (Age studies as cultural studies. Beyond the slice-of-life). Debate Feminista, 42, 79-108. https://www.jstor.org/stable/42625165

Hareven, T. K. (1996). Aging and Generational Relations: Life-Course and Cross-Cultural Perspectives. Walter de Gruyter.

Hernández, R., Fernández, C., \& Baptista, P. (2010). Metodología de la investigación (Research methodology). Mc Graw Hill.

Iacub, R. (2008). Estéticas de la existencia: ¿La vida es bella en la vejez? (Aesthetics of existence: Is life beautiful in old age?). Perspectivas en Psicología, 5(2), 10-18.

Losada-Baltar, A. (2004). Edadismo: consecuencias de los estereotipos, del prejuicio y la discriminación en la atención a las personas mayores. Algunas pautas para la intervención (Technologies of Aging: The Intersection between Feminist Film Theory and Age Studies). Informes Portal Mayores, (14). http://envejecimiento.csic.es/documentos/documentos/losada-edadismo-01.pdf

Medina, R. \& Zecchi, B. (2020). Tecnologías de la edad: La intersección entre teoría fílmica feminista y estudios etarios (Technologies of Age: The Intersection between Feminist Film Theory and Aging Studies). Investigaciones feministas, 11(2,) 251-262.

https://doi.org/10.5209/infe.66086

Meisner, B. A. (2020). Are You OK, Boomer? Intensification of Ageism and Intergenerational Tensions on Social Media Amid COVID-19. Leisure Sciences, 43(1-2), 56-61. https://doi.org/10.1080/01490400.2020.1773983

Méndez, L. (2020, May 4). Hora 25 (radio broadcast). Cadena SER. https://play.cadenaser.com/audio/cadenaser_hora25_20200504_220000_230000/

Montaña Blasco, M., Ollé Castellà, C., \& Lavilla Raso, M. (2020). Impacto de la pandemia de Covid-19 en el consumo de medios en España (Impact of the Covid-19 pandemic on media consumption in Spain). Revista Latina de Comunicación Social, (78), 155-167. https://www.doi.org/10.4185/RLCS-2020-1472

Muñoz, I. (2005). Los estereotipos de la vejez: Un problema social en vías de superación (The Stereotypes of the Age: A Social Problem in Vías of Superation). Pedagogía y saberes, (22), 9-20. https://doi.org/10.17227/01212494.22pys9.20

Muyor Rodríguez, J. (2021). Encuadre mediático del impacto del COVID-19 en las residencias de mayores. Un análisis de contenido sobre la identificación y la gestión del problema (Media framing of the impact of COVID-19 in nursing homes. A content analysis of the identification and management of the problem). EHQUIDAD. Revista Internacional De Politicas De Bienestar Y Trabajo Social, (15), 47-76. https://doi.org/10.15257/ehquidad.2021.0003

Neeo.es (2020). Estudio general de medios (General Media Survey). neeo.es. https:// www.neeo.es/2020/12/01/tercera-oleada-del-estudio-general-de-medios-egm-dediciembre-2020/

Palmore, E. (1999). Ageism: Negative and positive. Springer. 
Palmore, E. (2001). The Ageism Survey: First findings. Gerontologist, 41(5), 572-575. https://doi.org/10.1093/geront/41.5.572

Prieto, A. (2020a, March 19). Solamente una vez (radio broadcast). Radio Nacional de España. https://www.rtve.es/play/audios/solamente-una-vez/solamente-vez-all-alba-vincerogeopolitica-dudas-medicas-opera-19-03-20/5542198/

Prieto, A. (2020b, April 17). Solamente una vez (radio broadcast). Radio Nacional de España. https://www.rtve.es/play/audios/solamente-una-vez/solamente-vez-risas-tertuliaplanes-para-fin-semana-17-04-20/5559589/

Ramos-Soler, I. \& Carretón-Ballester, M. C. (2012). Presencia y representación de las personas mayores en la publicidad televisiva: el caso español (Presence and representation of older people in primetime television advertising: the Spanish case). Revista Española de Geriatría y Gerontología, 47(2), 55-61. https://doi.org/10.1016/j. regg.2011.11.010

Red de Radio Universitaria de Colombia. (@redradioucolombia). (2020, June 19). I Encuentro Virtual RIU, Conversatorio cuarto, 18 de junio de 2020 (I RIU Virtual Meeting, Fourth Conversation, June 18, 2020) (Video). YouTube. https://www.youtube.com/watch?v=H8uVLdwij7w

Rey-Beltrán, G. (2004). Prácticas comunicativas y representación social de las edades en los medios (Communicative practices and social representation of ages in the media). In Periodismo y Comunicación para todas las edades (Journalism and Communication for all ages) (pp.139-157). Ministerio de Comunicaciones de Colombia.

Rodero, E. (2020). Radio: the medium that best copes in crises. Listening habits, consumption, and perception of radio listeners during the lockdown by the Covid-19. El profesional de la información, 29(3), 290-306. https://doi.org/10.3145/epi.2020.may.06

Rodero, E. \& Blanco, M. (2020). El papel de la radio en situaciones de crisis. Iniciativas en la pandemia del coronavirus (The role of radio in crisis situations. Initiatives in the coronavirus pandemic). Index. Comunicación, 10(3), 193-213. http://hdl.handle.net/10115/17292

Suárez-Serrano, Ch. (2016). El periodismo en los conflictos armados del siglo XXI: entre las nuevas tecnologías y las amenazas de siempre (Journalism in the armed conflicts of the 21st century: between new technologies and perpetual threats). Revista del Instituto Español de Estudios Estratégicos, (8), 17-39. http://revista.ieee.es/article/view/218

Túñez-López, M., Vaz-Álvarez, M., \& Fieiras-Ceide, C. (2020). Covid-19 and public service media: Impact of the pandemic on public television in Europe. Profesional de la información, 29(5). https://doi.org/10.3145/epi.2020.sep.18

United Nations. (2020, May). Policy brief: The impact of COVID-19 on older persons. United Nations. https://www.un.org/development/desa/ageing/wp-content/uploads/sites/24/2020/05/ COVID-Older-persons.pdf 


\section{SOBRE LOS AUTORES}

VIRGINIA GUARINOS, es Doctora en Ciencias del Espectáculo y en Comunicación Audiovisual. Profesora titular de Comunicación Audiovisual de la Universidad de Sevilla (España) y directora del grupo de investigación AdMira, de análisis de medios, imágenes y relatos audiovisuales para el cambio social, de la Junta de Andalucía, Gobierno Autonómico. Es especialista en narrativa audiovisual, narrativa radiofónica, estudios culturales, estudios de género y estudios etarios.

iD https://orcid.org/0000-0002-7270-0087

DANIEL MARTíN-PENA, es Doctor en Educación y Comunicación Audiovisual. Profesor contratado doctor del área de Comunicación Audiovisual y Publicidad de la Universidad de Extremadura (España) y miembro del grupo de investigación "Educación transformadora para una sociedad global y digital" (EduTransforma-T) de la Junta de Extremadura. Especialista en radio, medios universitarios, educomunicación y divulgación científica. 